\title{
Analysis of judgmental adjustments in the presence of promotions
}

\author{
Juan R. Trapero ${ }^{\mathrm{a}, *}$, Diego J. Pedregal ${ }^{\mathrm{a}}$, R. Fildes ${ }^{\mathrm{b}}$, N. Kourentzes ${ }^{\mathrm{b}}$ \\ ${ }^{a}$ Universidad de Castilla-La Mancha \\ Departamento de Administracion de Empresas, Ciudad Real 13071, Spain \\ ${ }^{b}$ Lancaster University Management School \\ Department of Management Science, Lancaster LA1 4YX, UK
}

\begin{abstract}
Sales forecasting is increasingly complex due to a range of factors, such as the shortening of product life cycles, increasingly competitive markets, and aggressive marketing. Often, forecasts are produced using a Forecasting Support System that integrates univariate statistical forecasts with judgment from experts in the organization. Managers then add information to the forecast, such as future promotions, potentially improving the accuracy. Despite the importance of judgment and promotions, papers devoted to studying their relationship with forecasting performance are scarce. We analyze the accuracy of managerial adjustments in periods of promotions, based on weekly data from a manufacturing company. Intervention analysis is used to establish whether judgmental adjustments can be replaced by multivariate statistical models when responding to promotional information. We show that judgmental adjustments can enhance baseline forecasts during promotions, but not systematically. Transfer function models based on past promotions information achieved lower overall forecasting errors. Finally, a hybrid model illustrates the fact that human experts still added value to the transfer function models.
\end{abstract}

Keywords: Demand forecasting, Judgmental adjustments, Promotions, Transfer function, Intervention analysis

\section{Introduction}

Demand forecasting at the SKU level relies on a particular type of a Decision Support System, known as a Forecasting Support System (FSS), (Fildes, Goodwin, \& Lawrence, 2006). This FSS

\footnotetext{
${ }^{*}$ Corresponding author.

Email addresses: juanramon.trapero@uclm.es (Juan R. Trapero), diego.pedregal@uclm.es (Diego J. Pedregal), r.fildes@lancaster.ac.uk (R. Fildes), n.kourentzes@lancaster.ac.uk (N. Kourentzes) 
integrates a univariate statistical forecasting approach (system forecast), which delivers a baseline forecast, with managerial judgment from forecasters in the organization.

Univariate forecasting methods are based on time series techniques that analyze past sales history in order to extract a demand pattern that is projected into the future (Makridakis, Wheelwright, \& Hyndman, 1998). One example of such techniques is the family of exponential smoothing methods (Gardner, 2006; Hyndman, Koehler, Ord, \& Snyder, 2008). This kind of technique is very well-suited to companies that handle numerous SKUs where forecasts must be made semiautomatically. Nonetheless, these methods have some weaknesses, the most important of which, for this paper, is that they are not able to include additional potentially relevant information, such as promotions. In fact, promotional campaigns aim to modify customer behaviour, in order to increase sales. These changes affect customer demand making univariate forecasting algorithms inadequate for predicting promotional sales, since they are based on previous demand patterns, which do not include promotional periods. In order to solve that problem and improve forecast accuracy, managerial adjustments are employed. In fact, Franses \& Legerstee (2009) presented a case study where about $90 \%$ of al cases were adjusted. Among the reasons for adjusting forecasts, Fildes \& Goodwin (2007) indicated the main drivers behind the judgemental adjustment of statistical forecasts to be promotional and advertising activity.

Given the important relationship between judgmental forecasting and promotions, the first objective of this work is to analyze the accuracy of judgmentally adjusted forecasts applied to promotional campaigns. As far as the authors are concerned, this is the first case study that employs organisational data to verify whether judgmental forecasts during promotional periods achieve lower forecasting errors than its statistical counterpart.

An alternative approach to the problem of promotional forecasts involves the use multivariate statistical models that include information on past promotions for formulating causal models based on multiple linear regression whose exogenous inputs correspond to the promotion features (price discounts, type of display, type of advertising, etc.), (Özden Gür Ali, Sayin, van Woensel, \& Fransoo, 2009; Cooper, Baron, Levy, Swisher, \& Gogos, 1999). With this aim, several Promotional Support Systems (PSS) have been developed with promising results, see SCAN*PRO in Leeflang, van Heerde, \& Wittink (2002), PromoCast ${ }^{T M}$ in Cooper et al. (1999), and CHAN4CAST by Divakar, Ratchford, \& Shankar (2005). Nonetheless, the results of these methods have not been 
compared to judgmentally adjusted forecasts provided by experts. Therefore, our second objective is to develop an enhanced and automatically identified Exponential Smoothing model with intervention analysis and transfer function terms operating on a dummy promotion variable. We can then compare that model forecasts with judgmentally adjusted forecast in order to assess the extent of improvement that multivariate models can provide. Weekly real data from a manufacturing company are used to illustrate our findings.

Our results show that judgmentally adjusted forecasts of promotional periods may improve system forecasts, though not systematically, because when adjustments are overly large, forecasting accuracy can be reduced. Interestingly, a transfer function model based on past promotions information achieve on average lower forecasting errors than either system or judgmentally adjusted forecasts. In addition, a hybrid model is developed based on the transfer function model and expert judgment, and provides the lowest error among the methods considered.

This article is organized as follows: Section 2 summarizes the literature on promotional modeling and the role of judgmental adjustments. Section 3 introduces the case study and carries out an exploratory data analysis. Section 4 explores the main sources of judgmental bias. Section 5 explores different causal models in an attempt to improve the judgmental forecasts. Section 6 proposes a hybrid statistical-judgmental model, combining the best elements of each approach and finally, main conclusions are drawn in Section 7.

\section{Literature review}

Judgmental forecasting is an active research area, with interest in the area growning over the last 25 years. It has been recognised that human judgment may lead to important benefits in terms of forecasting accuracy but that such judgement may also cause many biases (Lawrence, Goodwin, O'Connor, \& Önkal, 2006). It should be noted that the topic of managerial adjustments in the particular context of supply chain demand forecasting has been overlooked until recently (Fildes, Goodwin, Lawrence, \& Nikolopoulos, 2009). In order to determine which variables are responsible for such biases, Fildes et al. (2009) analyzed the sign and size of adjustments as drivers of bias. They found that negative judgmental adjustments, i.e. judgment-based adjustments which decrease the statistical forecasts, are based on more reliable information and consequently lead to more accurate forecasts than positive adjustments, although this does seem to depend on the choice 
of error measure (Davydenko, 2012). Also, relatively larger adjustments tended to produce greater improvements than smaller adjustments, although part of this effect depends on the particular error measures used (Davydenko, Fildes, \& Trapero, 2010).

In order to reduce the bias and make judgmental forecasting more efficient, a range of models of varying level of complexity have been developed in order to integrate statistical forecasts and managers' judgment. For instance, Blattberg \& Hoch (1990) weighted the importance of each approach equally. Fildes et al. (2009) suggested an optimal model based on linear regression that determined the weight of statistical and judgmental forecasting based on the sign of the adjustments. Such differences between positive and negative adjustments motivated the use of nonlinear models by Trapero, Fildes, \& Davydenko (2011), who employed State Dependent Parameter estimation methods. The results concluded that: i) negative adjustments could be modeled by linear models; ii) positive adjustments followed a non-linear pattern; and iii) the managerial weight should be different depending on the adjustment size.

This attempt to find the appropriate balance between statistical and judgmental forecast can help us to understand the biases introduced by forecasters (Goodwin, 2000, 2005). Unfortunately, even after finding an equation for integrating judgmental adjustments with the baseline forecasts mechanically, some implementation issues might arise. For instance, forecasters may be less motivating to adjust the forecast, and therefore put less effort into performing the task (Belton \& Goodwin, 1996); or they may attempt to preempt the corrections by modifying their adjustments. Furthermore, the origin of the biases can also change over time (Fildes et al., 2009). Therefore, in order for managerial adjustments to be beneficial for improving statistical forecasts, a reduction of the judgemental adjustments is usually recommended (Goodwin, 2005). Behind this tendency to over-adjust, there might be a trend for associating a false pattern to random noise. One possible way to reduce the number of judgmental adjustments made by forecasters is to use the available information more efficiently. Thus, if we are looking for a reduction in the number of adjustments, a potential solution would be to model the effects of promotions on sales forecasts, for those promotions where past information is available. The forecasts would then be adjusted to take into account factors which are excluded from this enhanced model.

The modelling of promotions to enhance sales forecasting is not a new idea. Various Decision Support Systems have been designed for such a task. Cooper et al. (1999) designed a promotion- 
event forecasting system (PromoCast ${ }^{T M}$ ) where historical data were used to build a type of regression model on the basis of 67 variables. Divakar et al. (2005) developed CHAN4CAST, a web-based decision support system for forecasting sales consumer packaged goods. Essentially, most of the literature is centered on comparisons between extrapolation and causal methods; see a literature review by Özden Gür Ali et al. (2009). These methods assume, to some extent, that extrapolation and causal methods improve on the results achieved with judgmental adjustments made by managers, but there has been no empirical work that tested this assumption. Therefore, the research literature regarding the accuracy of judgmental adjustments when managers are responding to promotional information is too limited. Whether there are accuracy gains from either causal or extrapolation methods over and above any improvements from managerial adjustments, remains a moot point.

\section{Case study}

Our data have been collected from a manufacturing company specialized in household products. The data comprises: i) shipments; ii) one-step-ahead system forecasts; iii) one-step-ahead adjusted or final forecasts; and iv) a dummy variable whose value is 1 if there is a promotion and 0 otherwise. Our aim is to investigate whether an advance knowledge that a SKU is to be promoted can be used to improve managerial forecasts.

The dataset contains 169 SKUs, with a total of 25,012 complete triplets that have been sampled weekly between October 2008 and July 2011. Basically, the final forecast produced by the company is the result of two sources of information (Fildes et al., 2009). On the one hand, there is a piece of software which provides the statistical system forecasts. On the other hand, the company forecasters meet with personnel in sales, marketing, and production to share pieces of information that cannot be included in the statistical model. Thus the previous system forecast is adjusted by this group in accordance with their information, leading to an agreed forecast, the final forecast.

Observations with shipment values, system forecast or final forecast equal to zero have been removed from the original dataset in order to be able to use percentage error measures. After removing those observations, our sample size is 18,096 cases.

Table 1 shows some descriptive statistics of the collected data. In order to compare the information corresponding to different SKUs, the considered variables sales, system forecast (SF) and 
Table 1: Descriptive statistics of the case study data

\begin{tabular}{|c|c|c|c|c|c|c|c|c|}
\hline & \multirow{2}{*}{\multicolumn{2}{|c|}{$\mathrm{N}$}} & \multicolumn{3}{|c|}{ Median } & \multicolumn{3}{|c|}{ MdAD } \\
\hline & & & Sales & $\mathrm{SF}$ & $\mathrm{FF}$ & Sales & $\mathrm{SF}$ & $\mathrm{FF}$ \\
\hline No promo & 16581 & $(92 \%)$ & 2.01 & 1.96 & 2.13 & 1.17 & 0.82 & 1.03 \\
\hline Promo & 1515 & $(8 \%)$ & 2.21 & 1.49 & 2.41 & 1.45 & 0.68 & 1.68 \\
\hline Overall & 18096 & $(100 \%)$ & 2.03 & 1.91 & 2.15 & 1.17 & 0.82 & 1.03 \\
\hline
\end{tabular}

final forecast (FF) have been normalized with respect to the sales standard deviation. This table provides the Median as a robust metric of the size and the Median Absolute Deviation (MdAD) as a dispersion measure of Sales, SF and FF. Each statistic has been split in Promo and No Promo depending whether any type of promotion is active. The first column $(N)$ indicates the number of observations in each case. Note that the number of observations that are subject to promotions represents just $8 \%$ of the whole sample. As it is expected the median of sales increases for promotional observations. Interestingly, the MdAD of sales is also higher for promotional observations.

\subsection{Research questions}

In this section, a descriptive analysis of the data is carried out using common error measures, to answer some key questions. The first question we consider when studying judgmental adjustments is:

Q1:Are judgmental forecasts more accurate than statistical forecasts when there are promotions?

Table 2 shows some common error measures for assessing the accuracy of the system forecast (SF) compared to final forecasts $(\mathrm{FF})$. To achieve that aim the mean absolute percentage error, $M A P E=\operatorname{mean}\left(\left|P E_{t}\right|\right)$ and the median absolute percentage error, MdAPE $=\operatorname{median}\left(\left|P E_{t}\right|\right)$ were chosen as accuracy measures, where $P E_{t}$ is the percentage error given by:

$$
P E_{t}=100\left(y_{t}-F_{t}\right) / y_{t}, \quad t=1, \ldots, N
$$

Here, $y_{t}$ stands for the actual value and $F_{t}$ is the forecast, both of them at time $t$. The MdAPE is a more robust implementation of MAPE in the presence of outliers, Fildes (1992). These measures are those most commonly used in practice (Hyndman \& Koehler, 2006) due to their simplicity 
Table 2: Mean of MAPE and MdAPE to assess the forecasting error

\begin{tabular}{lcccc} 
& \multicolumn{2}{c}{ Mean(MAPE) } & \multicolumn{2}{c}{ Mean(MdAPE) } \\
\cline { 2 - 5 } & SF & FF & SF & FF \\
\hline No promo & 52.8 & 58.1 & 30.7 & 30.7 \\
Promo & 59.1 & 97.4 & 47.1 & 49.9 \\
Overall & 52.2 & 60.6 & 31.8 & 31.3 \\
\hline
\end{tabular}

of interpretation and applicability to this particular type of dataset. The percentage errors of these forecasts are used to calculate the MAPE and MdAPE of each individual SKU across time, and these are afterwards aggregated to give dataset average figures, obtaining the Mean(MAPE), Mean(MdAPE) as overall error measures over all SKUs. Based on the overall performances (last row of Table 2), different conclusions are reached depending on the error measure selected. The SF Mean(MAPE) is less than that of the FF. Conversely, Mean(MdAPE) indicates that FF performs better than SF. Therefore we have contradictory results, as is common in the literature (Trapero et al., 2011). One possible explanation might be the presence of extreme absolute percentage errors that can affect Mean(MAPE), as it is more sensitive to outlying values than its median counterpart. These extreme values could originate from judgmental adjustments to account for certain promotional information.

In order to analyze the influence of promotions, the results in the last row of Table 2 have been broken down according to whether there is a promotion or not. The first row (No promo) shows the results when no promotion is applied and the second row (Promo) when there is a promotion. Table 2 shows that forecasting errors are higher in the presence of promotions. More importantly, FF forecasts, which represent managerial adjustments, lead to worse predictions than system forecasts (SF), according to both by Mean(MAPE) and Mean(MdAPE).

According to these results, managerial adjustments increase forecast errors rather than decrease them. In that case, how are the managers reducing the forecasting accuracy? The reason behind such a bad performance of FF may be that judgmentally adjusted forecasts appear to be subject to bias (Fildes et al., 2009). In order to explore that possibility, the following question is proposed:

Q2: Are judgmental forecast adjustments biased in the presence of promotions? 
Table 3: Mean of MPE and MdPE to assess the forecasting error bias

\begin{tabular}{lcccc}
\hline & \multicolumn{2}{c}{ Mean(MPE) } & \multicolumn{2}{c}{ Mean(MdPE) } \\
\cline { 2 - 5 } & SF & FF & SF & FF \\
\hline No promo & -21.6 & -34.5 & 2.5 & -6.2 \\
Promo & 8.7 & -67.1 & 30.1 & -16.2 \\
Overall & -18.1 & -36.3 & 5.3 & -5.9 \\
\hline
\end{tabular}

The forecasting error bias can be analysed by means of percentage error measures without taking absolute values. Such measures include the Mean Percentage Error (MPE) and Median Percentage Error (MdPE). The advantage of these measures is that it is possible to aggregate them across SKUs. Considering equation (1), a positive value of the percentage error means that actual values are higher than the forecasts, and vice versa.

The last row in Table 3 shows the overall biases of SF and FF. For the judgmental forecasts $(\mathrm{FF})$, both error measures are negative indicating a bias towards optimism. Note that an optimism bias has been found in literature before (Fildes et al., 2009; Trapero et al., 2011). However, the bias associated with SF is not clear, because Mean(MPE) is negative but Mean(MdPE) is positive. Therefore, we can only conclude that judgmental forecast are biased towards optimism. In that sense, such an optimism may be the consequence of a sales increase being expected by managers in promotional periods. In order to explore that possibility, the overall results in Table 3 are disaggregated depending on whether there are promotions or not. Analyzing SF performance in promotional periods, the actual values are larger than the forecasts, leading to positive values for the means of both MPE and MdPE. Recall that SF is based on exponential smoothing models that only consider past data, and is therefore unable to foresee higher level of sales due to promotions. This drives managers to try to correct it by adjusting the SF. However, as the means of both MPE and MdPE of FF show, their adjustments are too optimistic.

\section{Exploring the bias: sign and size of adjustments}

The results in the last section showed that judgmentally adjusted forecasts are biased. The literature suggests a range of factors that may explain this judgmental forecasting bias, such as 
Table 4: Mean of MAPE and MdAPE to assess the forecasting error according to the adjustment sign

\begin{tabular}{lllllll}
\hline \multirow{2}{*}{ Adjustments } & \multirow{2}{*}{$\mathrm{N}$} & \multicolumn{2}{c}{ Mean(MAPE) } & \multicolumn{2}{c}{ Mean(MdAPE) } \\
\cline { 4 - 7 } & & & $\mathrm{SF}$ & $\mathrm{FF}$ & $\mathrm{SF}$ & $\mathrm{FF}$ \\
\hline Positive & 10691 & $(59.1 \%)$ & 47.9 & 65.7 & 32.5 & 31.8 \\
Negative & 4144 & $(22.9 \%)$ & 69.0 & 60.6 & 33.2 & 32.0 \\
None & 3261 & $(18.0 \%)$ & 49.7 & 49.7 & 30.8 & 30.8 \\
\hline
\end{tabular}

the size and sign of adjustments (Fildes et al., 2009; Syntetos, Nikolopoulos, Boylan, Fildes, \& Goodwin, 2009; Trapero et al., 2011). For example, small adjustments are expected to be less effective than large adjustments (Fildes et al., 2009). Furthermore, positive adjustments, those which increase the system forecast, are often less accurate than negative adjustments (Fildes et al., 2009; Trapero et al., 2011). In this section we explore the accuracy of the SF and FF as they depend on the size and sign of adjustments. In addition, we also investigate those variables for observations that are subject to promotions.

\subsection{Sign of adjustments}

Table 4 shows the Mean(MAPE) and the Mean(MdAPE) according to the sing of the adjustments. The second column shows that positive adjustments are more frequent than negative ones (59.1\% against 22.9\%). Regarding positive adjustments, it is not totally clear which method is most accurate, since it depends on the error measure. However, negative adjustments do improve the forecasting accuracy. These conclusions agree with those found in the literature (Fildes et al., 2009; Trapero et al., 2011). Differences found between SF and FF accuracy found using the Mean(MdAPE) are more attenuated.

Since the accuracy of the forecasts depends on both promotions and adjustment sign, the next step is to identify the relation between both factors. Table 5 shows the Mean(MAPE) and the Mean(MdAPE) according to both the sign of the adjustments and whether there is a promotion. It can be seen that most of the promotion observations are subject to positive adjustments.

For positive adjustments, $\mathrm{FF}$ is less accurate than SF for promotional periods. On the other hand, for non-promotional observations, the results achieved by Mean(MdAPE) indicate that FF is more accurate than SF. In addition, negative adjustments improve the forecasting accuracy 
Table 5: Mean of MAPE and MdAPE to assess the forecasting error depending on adjustment sign and whether it is subject to promotions

\begin{tabular}{llllcccc}
\hline \multirow{2}{*}{ Adjustments } & \multicolumn{2}{c}{$\mathrm{N}$} & \multicolumn{2}{c}{ Mean(MAPE) } & \multicolumn{2}{c}{ Mean(MdAPE) } \\
\cline { 5 - 8 } & & & $\mathrm{SF}$ & $\mathrm{FF}$ & $\mathrm{SF}$ & $\mathrm{FF}$ \\
\hline \multirow{2}{*}{ Positive } & Promo & 1245 & $(6.9 \%)$ & 54.1 & 104.0 & 46.9 & 52.7 \\
& No Promo & 9446 & $(52.2 \%)$ & 47.6 & 61.8 & 31.4 & 32.0 \\
\hline \multirow{2}{*}{ Negative } & Promo & 185 & $(1 \%)$ & 71.4 & 68.6 & 54.0 & 53.4 \\
& No Promo & 3959 & $(21.9 \%)$ & 68.6 & 59.7 & 32.8 & 31.6 \\
\hline \multirow{2}{*}{ None } & Promo & 85 & $(0.5 \%)$ & 71.4 & 71.4 & 66.9 & 66.9 \\
& No Promo & 3176 & $(17.6 \%)$ & 49.9 & 49.9 & 31.6 & 31.6 \\
\hline
\end{tabular}

provided by SF in both situations. Finally, it should be noted that there are only few promotions that have not been judgementally adjusted.

\subsection{Size of adjustments}

In our dataset there are SKUs with a range of different sales levels and variabilities. Thus, it is convenient to provide a framework within which to compare them. This can be done by means of data normalization. In particular, each product can be normalized with respect to its sales standard deviation, which can be interpreted as measuring the intrinsic difficulty of forecasting the particular SKU. Note that other normalization alternatives are possible. The SKU sales standard deviation has been chosen as a normalization factor here, in order for our results to be comparable with those of previous published works (Fildes et al., 2009; Trapero et al., 2011).

Table 6 shows the forecasting errors measured by the normalized MAE ${ }^{1}$. It should be pointed out that most of the MAE values are less than one, which means that forecast error variability is less than the sales variability. However, this does not hold for promotional periods. This is an indicator of the difficulty associated with forecasting promotional sales. During non-promotional periods, negative adjustments improve forecasting accuracy whereas positive adjustments do not. On the other hand, thw errors in promotional periods reveal larger errors for FF than for SF, for both positive and negative adjustments.

\footnotetext{
${ }^{1}$ The normalized MAE is referred to as MAE hereafter, since it is always used on normalized data in this paper.
} 
Table 6: MAE to assess the forecasting error depending on adjustment sign, size and whether it is subject to promotions

\begin{tabular}{lcccccc}
\hline & \multicolumn{2}{c}{ Overall } & \multicolumn{2}{c}{ Promotions } & \multicolumn{2}{c}{ No promotions } \\
\cline { 2 - 7 } Adjustments & SF & FF & SF & FF & SF & FF \\
\hline Positive & 0.80 & 0.86 & 1.19 & 1.40 & 0.75 & 0.78 \\
Negative & 0.72 & 0.69 & 1.11 & 1.22 & 0.70 & 0.66 \\
None & 0.66 & 0.66 & 0.74 & 0.74 & 0.66 & 0.66 \\
Total & 0.76 & 0.78 & 1.15 & 1.34 & 0.72 & 0.73 \\
\hline
\end{tabular}

One advantage of normalizing the dataset is that all the observations can be treated as crosssectional data. This transformation allows us to sort the data as a function of the size and sign of the adjustments. A useful visual interpretation can be obtained by plotting the MAE versus normalized adjustments, see Fig. 1. In this figure, the MAE has been separated based on the adjustment sign. For positive adjustments, it is interesting to note that FF is more accurate than SF when normalized adjustments are lower than approximately 3, and SF better for bigger adjustments. In the same figure, we also provide a histogram of the normalised adjustments by size, indicating that most of the adjustments are small, and the majority of them are positive.

Regarding negative adjustments some conclusions can be extracted from Table 6 and Fig. 1 : i) in general, MAE is lower than for positive adjustments; ii) on average, negative adjustments are smaller than positive ones; for instance, the maximum negative adjustment is around -2.5 , whereas positive adjustments can reach values close to 10 (i.e, adjustments can be 10 times higher than the variability of shipments for that SKU); and iii) on average, FF gives a lower error than SF.

One explanation for the improvement achieved by judgemental forecasting related to positive adjustments is the fact that managers know when there are promotions and can increase the values provided by the SF. In order to verify the managers judgment when there are promotions, Fig. 2 depicts the MAE obtained for positive and negative adjustments in the presence of promotions. Here, we can see clearly that positive adjustments improve on SF when they are lower than approximately 3. Furthermore, larger positive adjustments do not improve on the SF results, while smaller adjustments do. Negative adjustments in the presence of promotions tend to yield worse results than SF; however, they have less of an impact than positive adjustments, because they are 

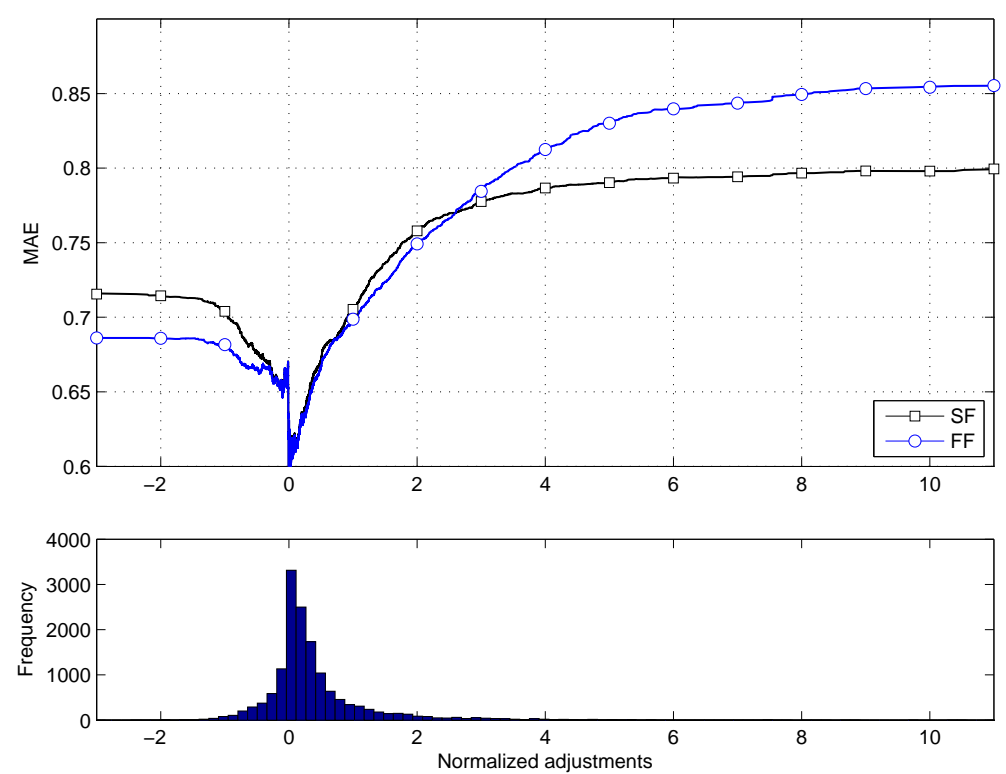

Figure 1: MAE vs. normalized adjustments, with a matching histogram.

less frequent and smaller, as it is shown in its histogram. It is interesting to note that most of the positive adjustments are located between 0 and 3 , which is the range of normalized adjustments where FF performs better than SF. This means that, in general, adjustments improve forecasting accuracy, but that a few large positive adjustments reduce the average accuracy of FF.
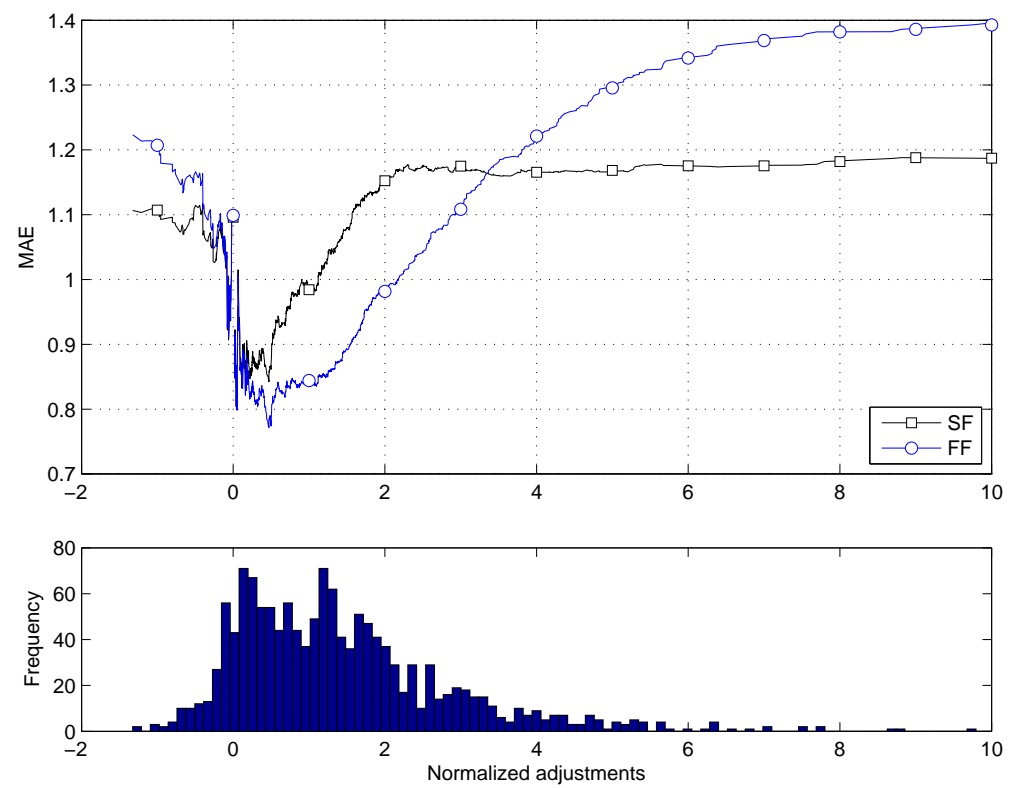

Figure 2: MAE vs. normalized adjustments with promotions, with a matching histogram. 
Finally, Fig. 3 shows the MAE vs. normalized adjustments and its histogram when there are no promotions. Essentially, positive adjustments do not improve SF significantly. The good performance of negative adjustments to FF relative to the original SF should be noted.
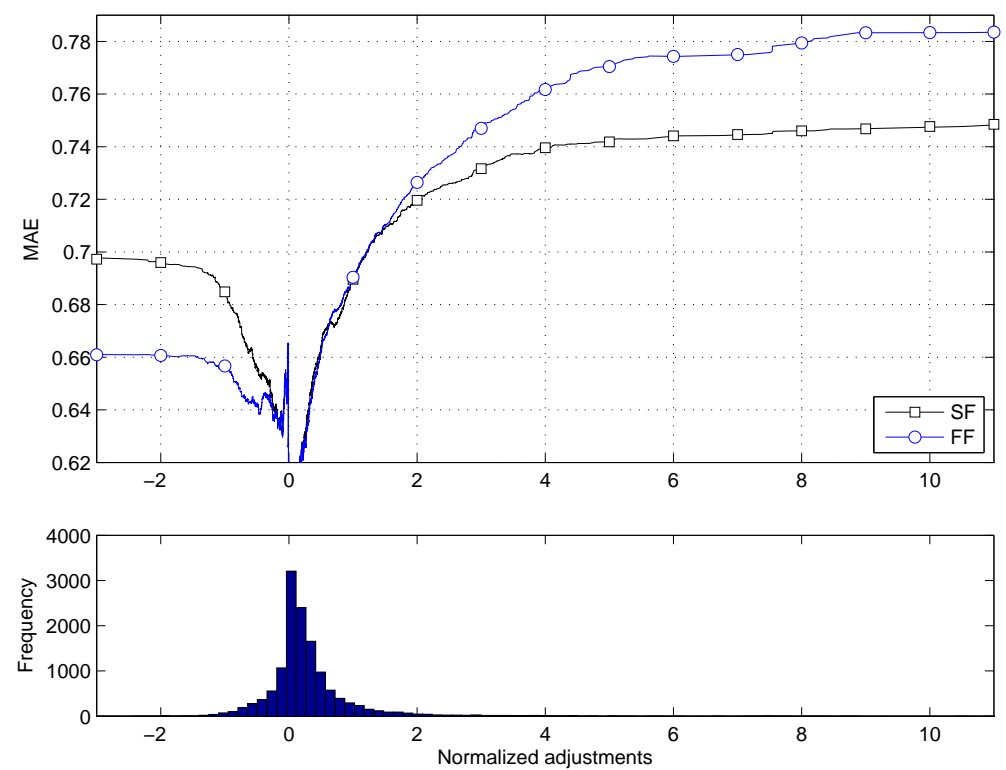

Figure 3: MAE vs. normalized adjustments without promotions, with a matching histogram.

In summary, positive adjustments are both larger and more frequent than negative adjustments. Moreover, while positive adjustments may improve forecast accuracy when there are promotions, this improvement is reduced as the adjustments get larger. Finally, negative adjustments when there are no promotions reduce the error considerably. This is probablly due to the fact that the managers know when a promotion is finished and adjust the system forecast back to its prepromotion levels.

\section{Promotional models}

In the previous section we discussed the fact that managers can adjust their system forecasts to include information about promotions that the System Forecast is unable to process, which has the potential to improve the forecasting accuracy; see Fig. 2. This result poses the following question: could the managers have obtained valuable information from a systematic analysis of past promotions? In other words, could the managers have analyzed patterns of past promotions and tried to project the results for forecasting similar future product promotions? In this case, 
multivariate statistical models could replace managers' adjustments, since human minds are not well suited to coping consistently with lots of information (Lawrence et al., 2006), as in this case study.

We now propose a simple approach for testing whether managerial adjustments can be replaced by multivariate models when dealing with promotions. This method is based on transfer function models operating on dummy variables that indicate whether there is a promotion or not. Moreover, that transfer function is combined with Exponential Smoothing models. The structure of the transfer function is identified automatically by the Schwarz Information Criterion (Schwartz, 1978).

\subsection{Automatically identified Transfer Function}

The simplest version of the model is an Exponential Smoothing model for the non promotions situations, as follows:

$$
\begin{aligned}
& y_{t}=l_{t-1}+e_{t} \\
& l_{t}=l_{t-1}+\alpha e_{t}
\end{aligned}
$$

where $l_{t}$ is a time varying level of sales and $e_{t}$ is a white noise sequence with zero mean and constant variance.

The model is expanded with a transfer function term that operates on a dummy variable consisting of a step for the samples where the promotion is activated:

$$
\begin{aligned}
& y_{t}=l_{t-1}+\frac{B(L)}{A(L)} P_{t}+e_{t} \\
& l_{t}=l_{t-1}+\alpha e_{t}
\end{aligned}
$$

where $L$ is the backshift operator such that $L^{j} y_{t}=y_{t-j}, B(L)=\left(b_{0}+b_{1} L+b_{2} L^{2}+\ldots+b_{m} L^{m}\right)$ is a polynomial in the backward shift operator of order $m, A(L)=\left(1+a_{1} L+a_{2} L^{2}+\ldots+a_{n} L^{n}\right)$ is a polynomial of order $n$ and $P_{t}$ is a binary dummy variable with ones in the weeks where there is a promotion. The timings and lengths of promotions are known in advance.

Prior to the estimation of the transfer function, the specific orders of the numerator and denominator polynomials have to be identified. In this particular case, this is achieved by minimising the well known Schwartz Information Criterion on a range of possible models. The models include combinations of polynomials for the numerator of orders one to five and zero to one in the denominator. 
The amount of information used for the identification of the transfer function models differs depending on the situation. For SKUs with only one promotion or the first promotion of SKUs with several promotions, no prior information is available, and the model has to be identified dynamically. In SKUs where several promotions are implemented, the model takes advantage of the immediate past promotion; i.e. the model identified for the previous promotion is used as a starting point for the next, and all the information about the previous promotion is included in the identification stage for the next.

This case study is based on one-step-ahead forecasts (where the step size is a week). It implies that all models are always estimated using information available up to the forecast origin in order to produce the best forecasts possible. This means that as a promotion advances in real time, different transfer function orders and/or different parameters are used for each of the forecasts produced. All models are estimated by Exact Maximum Likelihood using the ECOTOOL Matlab toolbox, see Pedregal, Contreras, \& Sanchez (2010).

\subsection{Experimental setup}

In sections 3 and 4 , the whole dataset was used to analyze the accuracy of the system and final forecasts given by the company. In this section that dataset is split to carry out a predictive empirical evaluation experiment. Since the number of observations with and without promotions are different, we have separated the sample in the following way: i) where enough data is available, the estimation sample for determining the coefficient of the exponential smoothing costintutes $50 \%$ of the data on non-promoted observations; and ii) given that only $8 \%$ of the whole dataset is affected by promotions, all those observations are considered as part of the hold-out sample, i.e., all the promotions will be forecast.

In order to determine whether the Transfer Function (TF) model proposed is able to reduce promotional forecasting errors, Fig. 4 depicts the MAE associated to the System Forecast (SF), Final Forecast (FF) and Transfer Function model (TF) as a function of the normalized judgmental adjustments on the hold-out sample. It is important to note that the TF model is capable of capturing part of the knowledge that experts have included in their forecast when adjustments are not too large, as well as avoiding large FF errors in situations when large adjustments were made. In that sense, the TF model achieves the lowest error of the three approaches on average. These results suggest that adjustments applied to promotions can be substituted by statistical models 
obtaining lower forecasting errors and reducingmanagers' workload when judgmentally analysing and adjusting system forecasts.
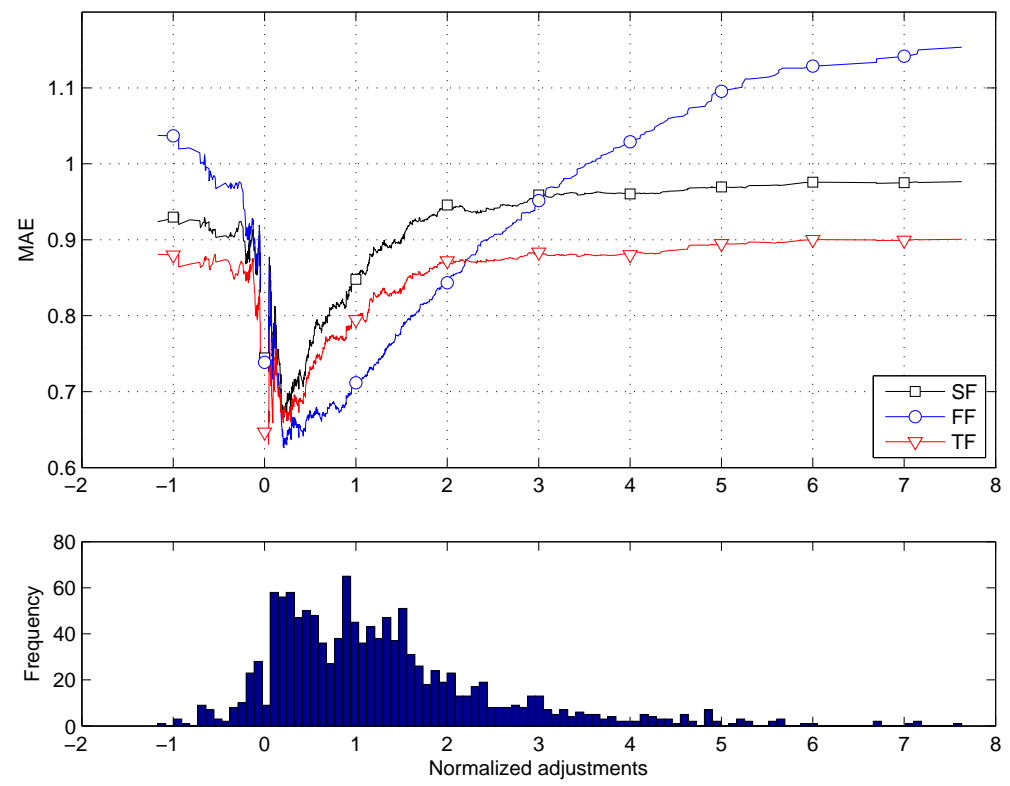

Figure 4: MAE of normalized adjustments with promotions on the hold-out sample

Figure 5 shows the three forecasts considered in the absence of promotions. It can be seen that negative adjustments in FF considerably reduce the forecasting errors. This result agrees with the findings of previous studies (Fildes et al., 2009; Trapero et al., 2011) where forecasters achieved better forecasts when making negative adjustments, because their information might be more realistic than promotional information. In addition, TF forecasts are more accurate than $\mathrm{SF}$ ones. A possible explanation for this is that SF forecasts do not distinguish promotional and non-promotional periods, meaning that after several promotional weeks, the univariate algorithm implemented in SF will take some time to return to the average non-promotional sales. In other words, if algorithms that yield baseline forecasts are univariate, and therefore do not distinguish promotional periods, their optimization procedures may provide biased parameter estimates. This may result in a reduction in their forecasting accuracy over promotional weeks, as well as the previous and subsequent weeks, given the dynamic nature of promotions.

In cases of positive adjustments, adjustments generally also achieve lower forecasting errors. However, the presence of adjustments whose size is greater than four (when normalised by the SKU standard deviation) means that FF performs worse on average than either SF or TF. 

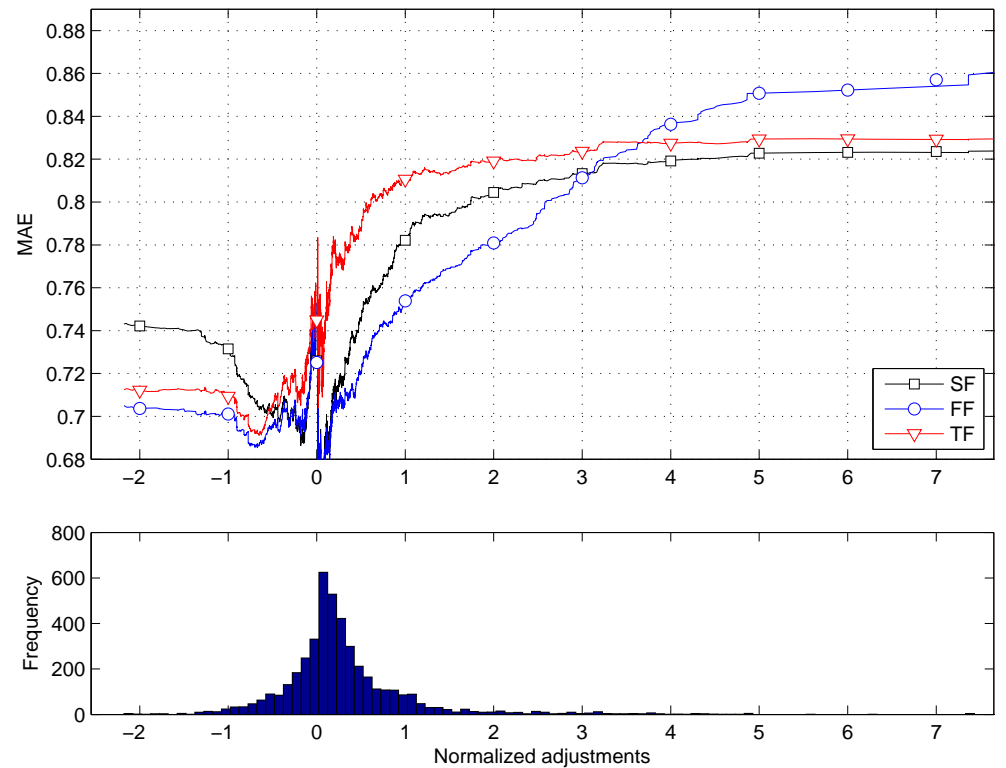

Figure 5: MAE of normalized adjustments without promotions on the hold-out sample

\subsection{Experimental results}

Table 7 shows the forecasting errors on the hold-out dataset achieved by the SF, FF and TF (Transfer Function) models. The first two rows show the percentage errors Mean(MdAPE) and Mean $(\mathrm{MdPE})^{2}$, and the third row shows the MAE on the normalized hold-out sample. Essentially, all measures agree that SF performance is better than FF overall, as a consequence of the greater forecasting errors made during promotions. Moreover, Mean(MdPE) indicates that judgmental adjustments included in FF are biased towards optimism, particularly when there are promotions.

In relation to the TF accuracy, TF performs better than $\mathrm{SF}$ and $\mathrm{FF}$. It is interesting to point out the good results obtained by TF during promotions. Moreover, since TF works as an exponential smoothing model during non promotional periods, TF and SF produce similar errors when there are no promotions.

\subsection{An alternative error measure: ARMAE}

Percentage errors as Mean(MdAPE) and Mean(MdPE) together with MAE on normalized data have been used previously to measure the accuracy of judgmental forecasts and the influence of

\footnotetext{
${ }^{2}$ Note that other possible percentages error measures, such as Mean(MAPE) and Mean(MPE), have not been included in Table 7 because they might lead to a misunderstandings, given that they are less robust to extreme values than their versions based on the median (Mean(MdAPE) and Mean(MdPE)), as explained earlier.
} 
Table 7: Forecasting errors of SF, FF and TF on the hold-out dataset. The error measures chosen are Mean(MdAPE), Mean(MdPE), MAE and ARMAE

\begin{tabular}{lccccccccc}
\hline & \multicolumn{3}{c}{ Overall } & \multicolumn{4}{c}{ Promotions } & \multicolumn{3}{c}{ No promotions } \\
\cline { 2 - 10 } & SF & FF & TF & SF & FF & TF & SF & FF & TF \\
\hline Mean(MdAPE) & 38.6 & 40.0 & 35.8 & 47.7 & 50.7 & 43.1 & 27.6 & 26.9 & 26.9 \\
Mean(MdPE) & 19.2 & -10.3 & 9.5 & 32.0 & -16.8 & 14.1 & 3.6 & -2.3 & 3.9 \\
MAE & 0.819 & 0.858 & 0.805 & 0.956 & 1.119 & 0.886 & 0.786 & 0.797 & 0.787 \\
ARMAE & - & 1.04 & 0.97 & - & 1.14 & 0.93 & - & 1.01 & 1.00 \\
\hline
\end{tabular}

promotions on such errors. Nonetheless, even when the aforementioned error metrics are commonly accepted, Makridakis (1993) and Hyndman \& Koehler (2006) point out the limitations of percentage errors because they overweight the large errors which result when the actual value $y_{t}$ is relatively small compared to the forecast error.

In order to overcome those disadvantages of percentage errors, Hyndman \& Koehler (2006) proposed the MASE (mean absolute scaled error). However, MASE introduces a bias towards overrating the performance of a benchmark forecast as a result of arithmetic averaging (Davydenko et al., 2010). To avoid that overrating, Davydenko et al. (2010) proposed the Average Relative Mean Absolute Error (ARMAE) based on a geometric average. Here, the system forecast is employed as benchmark, i.e:

$$
A R M A E=\left(\Pi_{i=1}^{m} r_{i}^{n_{i}}\right)^{1 / \sum_{i=1}^{m} n_{i}}, \quad r_{i}=\frac{M A E_{i}^{a}}{M A E_{i}^{b}}
$$

where $M A E_{i}^{b}$ is the MAE for baseline statistical forecast, and $M A E_{i}^{a}$ is the MAE for the alternative forecasting methods. The alternatives considered are: i) the judgmentally adjusted forecast (FF); and ii) the transfer function forecast (FF). Both are computed for each SKU $i . n_{i}$ stands for the number of available errors for the $i^{\text {th }} \mathrm{SKU}$ and $m$ is the number of SKUs under study. MAEs in Eq. (4) are computed as: 


$$
\begin{aligned}
& M A E_{i}^{a}=\frac{1}{n_{i}} \sum_{t \in T_{i}}\left|e_{i, t}^{a}\right| \\
& M A E_{i}^{b}=\frac{1}{n_{i}} \sum_{t \in T_{i}}\left|e_{i, t}^{b}\right|
\end{aligned}
$$

where $e_{i, t}^{a}$ and $e_{i, t}^{b}$ represent the errors for the alternative forecasts and the baseline statistical forecasts, respectively. $T_{i}$ is a set containing time periods for which $e_{i, t}^{a}$ are available. ARMAE can be interpreted as follows: values of $A R M A E<1$ indicate that, on average, $M A E_{i}^{a}<M A E_{i}^{b}$ and consequently the alternative methods performs better the than baseline forecasts.

The last row in Table 7 shows the ARMAE achieved by the various methods we have discussed. It agrees with the rest of error measures that adjustments (FF) do not improve forecasting accuracy when there are promotions. More importantly, the ARMAE agrees with Mean(MdAPE), Mean(MdPE) and MAE that the TF model proposed delivers the lowest forecasting error, and shows that TF also achieves a significant error reduction when there are promotions.

\section{A Hybrid model to forecast promotional sales}

The previous section showed that TF can reduce the forecasting error on promoted weeks considerably. Nonetheless, Fig. 4 also shows that FF might achieve the lowest forecasting error for an interval of normalized adjustments between 0 and 2.5. It is interesting to assess whether the managers' judgmental adjustments still contain useful information for TF. Focusing on the sample where $\mathrm{TF}$ forecasts are available and there are promotions, a forecast encompassing test (Fang, 2003) is carried out:

$$
y_{t}=\beta_{0}+\beta_{1} F F_{t}+\beta_{2} T F_{t}+e_{t},
$$

where $\beta_{1}$ and $\beta_{2}$ are constrained to add up to 1 and $\beta_{0}$ allows for the possibility of bias. A significant coefficient of the FF model implies that it captures additional information, which is currently missing from TF. We find that this is the case, with a p-value less than 0.01.

Therefore, a model that could potentially improve the results could be the combination of TF and FF. In this sense, a hybrid model in terms of a linear regression is defined such as:

$$
y_{t}=\alpha_{0}+\left(\alpha_{1} T F_{t}+\alpha_{2} F F_{t}\right)+\left(\alpha_{3} T F_{t}+\alpha_{4} F F_{t}\right) X_{1}+\epsilon_{t}
$$


where $\alpha_{0}$ in stands for the bias and $\epsilon_{t}$ is the error term. $X_{1}$ is a dummy variable that allows the weights of $\mathrm{TF}$ and FF to vary depending on the adjustments size, i.e:

$$
X_{1}=\left\{\begin{array}{l}
0 \text { if } 0 \leq \text { adjustments } \leq 2.5 \\
1 \text { otherwise }
\end{array}\right.
$$

In order to evaluate the hybrid model performance, the following experiment is designed. Let the total sample be determined by those observations where TF forecasts are available and there are promotions. Then, that sample is divided into two parts: firstly, an estimation sample, which comprises $60 \%$ of the total sample, and secondly, the hold-out sample with the rest of observations.

The results of the estimated hybrid model applied to the hold-out sample can be seen in Fig. 6, where the hybrid model provides the lowest error. We compare this model with the rest of methods as well as the 50\%-50\% model (BH) defined by Blattberg \& Hoch (1990). We can see that the hybrid model produces the lowest errors by combining the two sources of information, indicating that the managers' adjustments still contain useful information.
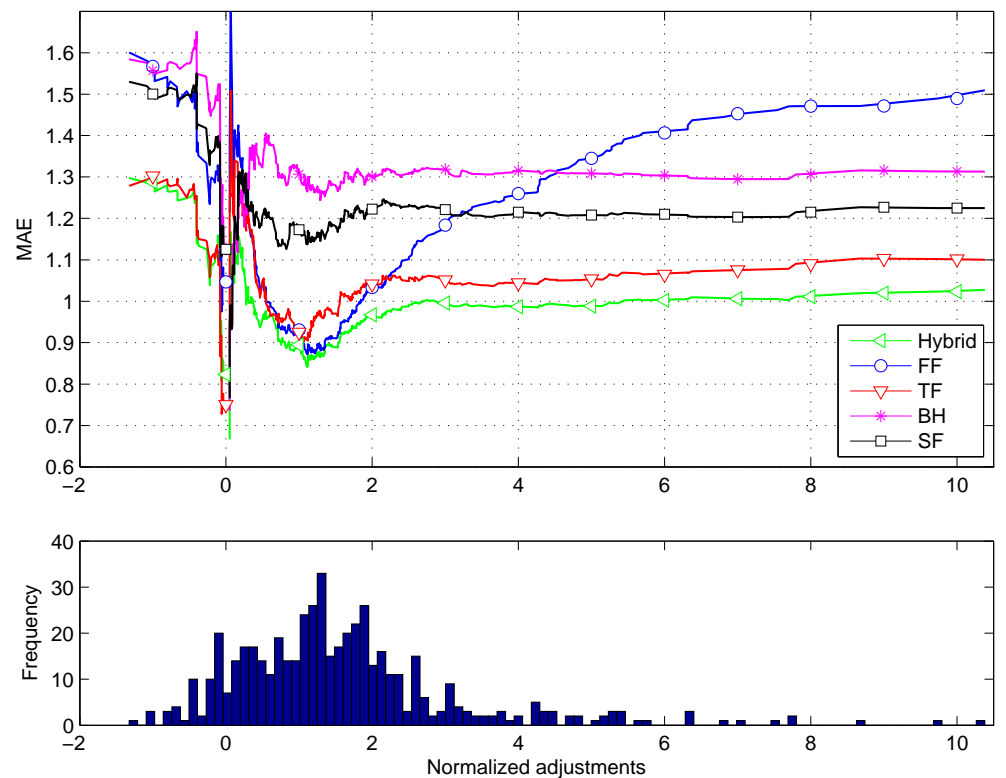

Figure 6: MAE of considered models vs. normalized adjustments. Histogram of normalized adjustments

It is questionable how applicable is such a model in practice. It assumes that the judgmental adjustments will happen the same way, even if experts know that their forecasts will be consequently combined with a statistical model, which is not to be expected. However, it provides evidence that combining statistical models of promotions with judgmental information can lead to substantial 
gains in accuracy. Furthermore, it illustrates that experts add value to the forecasting process, even if we move away from simple baseline forecasting models to promotional models, as in the TF.

\section{Conclusions}

Judgmental forecasting is commonly employed to modify system forecasts when promotions are taking place in order to achieve a lower forecasting error. Interestingly, little empirical research has been done to analyze the efficiency of these adjustments. This paper investigates the accuracy of judgmental forecasting at SKU level in the presence of promotions. The first finding is that judgmentally adjusted forecasts may enhance forecasts under certain circumstances. The analysis of the sign and size of the adjustments has been shown to be crucial to understanding the conditions that lead to better forecasts. A superficial exploratory data analysis could lead to the conclusion that experts' adjustments reduce the forecasting accuracy, which could therefore lead to the suggestion to eliminate judgmental forecasting in promotional periods. However, a deeper analysis concluded that when adjustments size was not too large, experts reduced forecasting error. In addition, a bias towards optimism was also found, since positive adjustments tended to lead to larger errors than negative ones.

In order to generalize those results, more empirical evidence from other companies and industries is required. For instance, it would be interesting to investigate whether the estimated sales uplift on promotional weeks shown in Table $3(\mathrm{Mean}(\mathrm{MPE})=8.7 \%$ and $\mathrm{Mean}(\mathrm{MdPE})=30.1 \%)$ is typical or not, and whether the size of such uplift may influence the precision of expert adjustments.

Since experts made their adjustments on the basis of an analysis of past promotional demand, an alternative is to replace judgmental forecasting with a mathematical model for forecasting promotional sales. This research has presented a simple model based on a transfer function, automatically identified, combined with single exponential smoothing. The aim of this model was not to provide optimal forecast, but to show that if a simple model could beat judgmental forecasting, investing more resources and effort to develop a sophisticated model would be a worthwhile. The results showed that, on average, this simple model could perform better than the expert adjustments.

There are three main advantages to modelling promotions. Firstly, experts can benefit from a reduction in the effort required to judgmentally adjust forecasts. Secondly, more accurate forecasts 
can be achieved. Finally, modelling promotional effects can also improve accuracy when forecasting for non-promotional periods, since unusually high sales data, as a consequence of a promotion, lead to overly high forecasts in subsequent periods, after the promotion is ended.

Given the potential benefits of mathematical modelling, further research should investigate different issues that can arise. For instance, i) how many promotional variables should be stored and the cost of this; ii) how should we choose the selection method among the potential promotional variables; iii) how should the selected variables be incorporated efficiently into more sophisticated methods, ranging from linear to non-linear models; and finally iv) how can these models can be implemented by companies with a possible lack of expertise in handling such complex techniques.

Finally, a simple hybrid model that integrated judgmental adjustments and the transfer function forecasts showed that experts still added value to the forecasts. However, one limitation of such endeavours is that our understanding of the way in which experts will change their adjustments in the light of a forecasting support system that adjusts further their forecasts is very limited.

\section{Acknowledgment}

The authors would like to acknowledge the support provided by The International Institute of Forecasters and SAS research grant and the Junta de Comunidades de Castilla-La Mancha project number PII1I09-0209-6050

\section{References}

Belton, V. \& Goodwin, P. (1996). On the application of the analytic hierarchy process to judgmental forecasting. International Journal of Forecasting, 12, 155-161.

Blattberg, R. C. \& Hoch, S. J. (1990). Database models and managerial intuition: 50\% model $+50 \%$ manager. Management Science, 36, 887-899.

Cooper, L. G., Baron, P., Levy, W., Swisher, M., \& Gogos, P. (1999). PromoCast trademark: A new forecasting method for promotion planning. Marketing Science, 18(3), 301.

Davydenko, A. (2012). Integration of Judgmental and Statistical Approaches for Demand Forecasting: Models and Methods. Ph.D. thesis, Lancaster University.

Davydenko, A., Fildes, R., \& Trapero, J. R. (2010). Measuring the accuracy of judgmental adjustments to sku-level demand forecasts. Tech. rep., Lancaster University Management School Working Paper 2010/026.

Divakar, S., Ratchford, B. T., \& Shankar, V. (2005). CHAN4CAST: A multichannel, multiregion sales forecasting model and decision support system for consumer packaged goods. Marketing Science, 24, 334-350. 
Fang, Y. (2003). Forecasting combination and encompassing tests. International Journal of Forecasting, $19(1), 87$ 94.

Fildes, R. (1992). The evaluation of extrapolative forecasting methods. International Journal of Forecasting, 8, 81-98.

Fildes, R. \& Goodwin, P. (2007). Against your better judgment? how organizations can improve their use of management judgment in forecasting. Interfaces, 3\%, 70-576.

Fildes, R., Goodwin, P., \& Lawrence, M. (2006). The design features of forecasting support systems and their effectiveness. Decision Support Systems, 42(1), $351-361$.

Fildes, R., Goodwin, P., Lawrence, M., \& Nikolopoulos, K. (2009). Effective forecasting and jugdmental adjustments: an empirical evaluation and strategies for improvement in supply-chain planning. International Journal of Forecasting, 25, 3-23.

Franses, P. H. \& Legerstee, R. (2009). Properties of expert adjustments on model-based SKU-level forecasts. International Journal of Forecasting, 25, 35-47.

Gardner, E. S. (2006). Exponential smoothing: The state of the art, Part II. International Journal of Forecasting, 22, 637-666.

Goodwin, P. (2000). Correct or combine? mechanically integrating judgmental forecasts with statistical methods. International Journal of Forecasting, 16, 261-275.

Goodwin, P. (2005). How to integrate management judgment with statistical forecasts. Foresight: The International Journal of Applied Forecasting, 1, 8-12.

Hyndman, R. J. \& Koehler, A. B. (2006). Another look at measures of forecast accuracy. International Journal of Forecasting, 22, 679-688.

Hyndman, R. J., Koehler, A. B., Ord, J. K., \& Snyder, R. D. (2008). Forecasting with Exponential Smoothing: The State Space Approach. Berlin, Heidelberg: Springer-Verlag.

Lawrence, M., Goodwin, P., O'Connor, M., \& Önkal, D. (2006). Judgmental forecasting: A review of progress over the last 25 years. International Journal of Forecasting, 22, 493-518.

Leeflang, P. S., van Heerde, H. J., \& Wittink, D. (2002). How promotions work: SCAN*PRO-based evolutionay model building. Schmalenbach Business Review, 54, 198-220.

Makridakis, S. (1993). Accuracy measures: theoretical and practical concerns. International Journal of Forecasting, 9, 527-529.

Makridakis, S., Wheelwright, S. C., \& Hyndman, R. J. (1998). Forecasting: Methods and applications. Third Edition. John Wiley \& Sons.

Özden Gür Ali, Sayin, S., van Woensel, T., \& Fransoo, J. (2009). SKU demand forecasting in the presence of promotions. Expert Systems with Applications, 36(10), 12340 - 12348.

Pedregal, D. J., Contreras, J., \& Sanchez, A. (2010). Handbook of Networks in Power Systems, chap. ECOTOOL: A general MATLAB forecasting toolbox with applications to electricity markets, pp. 69-104. Springer Verlag.

Schwartz, G. (1978). Estimating the dimension of a model. Ann. Stat., 6, 461-464.

Syntetos, A. A., Nikolopoulos, K., Boylan, J. E., Fildes, R., \& Goodwin, P. (2009). The effects of integrating management judgement into intermittent demand forecasts. International Journal Production Economics, 118, 72-81. 
Trapero, J. R., Fildes, R., \& Davydenko, A. (2011). Nonlinear identification of judgmental forecasts effects at SKU level. Journal of Forecasting, 30, 490-508. 